\title{
Management approach for NASA's Earth Venture-1 (EV-1) airborne science investigations
}

\author{
Anthony R. Guillory", Todd C. Denkins, and B. Danette Allen \\ NASA Earth System Science Pathfinder (ESSP) Program Office, NASA Langley Research Center, \\ Hampton, VA
}

\begin{abstract}
The Earth System Science Pathfinder (ESSP) Program Office (PO) is responsible for programmatic management of National Aeronautics and Space Administration's (NASA) Science Mission Directorate's (SMD) Earth Venture (EV) missions. EV is composed of both orbital and suborbital Earth science missions. The first of the Earth Venture missions is EV-1, which are Principal Investigator-led, temporally-sustained, suborbital (airborne) science investigations costcapped at $\$ 30 \mathrm{M}$ each over five years. Traditional orbital procedures, processes and standards used to manage previous ESSP missions, while effective, are disproportionally comprehensive for suborbital missions. Conversely, existing airborne practices are primarily intended for smaller, temporally shorter investigations, and traditionally managed directly by a program scientist as opposed to a program office such as ESSP. In 2010, ESSP crafted a management approach for the successful implementation of the EV-1 missions within the constructs of current governance models. NASA Research and Technology Program and Project Management Requirements form the foundation of the approach for EV-1. Additionally, requirements from other existing NASA Procedural Requirements (NPRs), systems engineering guidance and management handbooks were adapted to manage programmatic, technical, schedule, cost elements and risk. As the EV-1 missions are nearly at the end of their successful execution and project lifecycle and the submission deadline of the next mission proposals near, the ESSP PO is taking the lessons learned and updated the programmatic management approach for all future Earth Venture Suborbital (EVS) missions for an even more flexible and streamlined management approach.
\end{abstract}

Keywords: Project management, Reviews, Earth Science, Systems Engineering, Remote sensing, Earth Venture

\section{INTRODUCTION}

The Earth System Science Pathfinder (ESSP) Program Office (PO) is responsible for programmatic management of National Aeronautics and Space Administration's (NASA) Science Mission Directorate's (SMD) Earth Venture (EV) orbital and suborbital (airborne) missions. Guidance for project lifecycle plans and processes required for the successful execution of these missions within the constructs of current governance models is a critical component of this responsibility. The first of the EV missions selected was EV-1, the largest agency suborbital effort of its kind to date. Proposals for Principal Investigator-led, temporally-sustained, airborne-science investigations were solicited and competitively-selected via a NASA Research Announcement [1]. On 27 May 2010, five investigations cost capped at no more than $\$ 30 \mathrm{M}$ each with a period of performance not to exceed five years were selected covering a broad range of Earth science focus areas [2].

The awarded investigations were selected based on scientific and technical merit with consideration for NASA's programmatic aim to contribute to as many Earth Science research focus areas as possible. The EV-1 investigations span the Earth science focus areas of atmospheric composition, weather, climate variability and change, water and energy cycles, and carbon cycle and ecosystems and include Earth science researchers from NASA as well as other government agencies, academia and industry from around the world. The EV-1 Principal Investigators (PIs) and investigations are Dr. Mahta Moghaddam, University of Southern California [Airborne Microwave Observatory of Subcanopy and Subsurface (AirMOSS)]; Dr. Eric Jensen, NASA Ames [Airborne Tropical Tropopause Experiment (ATTREX)]; Dr.

\footnotetext{
* anthony.r.guillory@nasa.gov; phone 1757 864-8655; fax 1757 864-8435; http://science.nasa.gov/about-us/smdprograms/earth-system-science-pathfinder/
} 
Charles Miller, NASA Jet Propulsion Laboratory [Carbon in Arctic Reservoirs Vulnerability Experiment (CARVE)]; Dr. James Crawford, NASA Langley [Deriving Information on Surface Conditions from Column and Vertically Resolved Observations Relevant to Air Quality (DISCOVER-AQ)]; and Dr. Scott Braun, NASA Goddard [Hurricane And Severe Storm Sentinel (HS3)].

There are many aspects of EV-1 that differentiate it from previous airborne science investigations, which, in turn demands a change in the management approach. The EV-1 airborne-science investigations are broader in scope than typical airborne-science missions due to multiple deployments, sustained science data collection and operations spanning several years. Furthermore, the EV-1 investigations need to satisfy a set of verifiable Program Level Requirements that are agreed to at the NASA Headquarters (HQ) Earth Science Division (ESD) level and are cost capped whereas previous airborne investigations that had documented goals with less formality without a defined cost cap. Another factor that differentiates EV-1 is that these investigations have increased visibility within NASA and outside of NASA which drives the reporting requirements. On EV-1, the PI's are fully responsible for the entire investigation whereas in past airborne investigations the Program Scientist at HQ was responsible for all aspects of the mission, including execution. Therefore, a tailored approach to mission management has been crafted that accommodates the risk-tolerant, real-time operational approach of airborne-science while maintaining a level of rigor commensurate with Earth science missions of this magnitude. Policy and best practices from existing NASA Procedural Requirements (NPRs), systems engineering guidance [3], management handbooks [4] and center processes were adapted in order to manage the programmatic, technical, schedule, cost and risk elements of the EV-1 airborne science investigations. Traditional procedures, processes and standards used to manage Space Flight [5] and Research and Technology [6] represent proven NASA best practices and were leveraged to determine the gates and major events for each Life Cycle Phase, with an emphasis on established Research and Technology management requirements [6]. Consideration for the high Technology Readiness Level (TRL) required of the proposals allowed for streamlining of the formulation phase of the project life cycle. This enables EV-1 investigations to enter into operations as quickly as possible in order to maximize the acquisition of science data. Additionally, an iterative operational phase allows for multiple deployments over multiple years and retains the ability to incorporate design and operational advancements.

The EV-1 missions bring together various communities of stakeholders to manage and implement these Earth science investigations. These communities include the ESSP Program, NASA's Airborne Science Program, NASA's Applied Sciences Program, six NASA centers, NASA Headquarters, and Earth science researchers from government agencies, academia, and industry around the world. The mission management approach presented here is currently utilized for EV1 and will set the precedent for future Earth Venture airborne-science missions.

\section{PREVIOUSLY SELECTED ESSP MISSIONS}

Historically, the ESSP Program has been characterized by relatively low to moderate cost, small to medium sized orbital missions that are capable of being built, tested, and launched in a relatively short time interval. These missions supported a variety of scientific objectives related to Earth science, including the atmosphere, oceans, land surface, polar ice regions, and solid-Earth. Investigations include development and operation of remote sensing instruments and the conduct of investigations utilizing data from these instruments. [7] ESSP's first Announcement of Opportunity (AO) was released in 1996 and the Program Office's long history has resulted in many successful missions including Gravity Recovery and Climate Experiment (GRACE) [8], CloudSat [9], and Cloud-Aerosol Lidar, Infrared Pathfinder Satellite Observations (CALIPSO) [10] and Aquarius/SAC-D mission [11]. The Orbiting Carbon Observatory (OCO) 2 [12] is in the development phase. The Orbiting Carbon Observatory (OCO) 3 is in formulation.

\section{VENTURE CLASS MISSIONS}

NASA initiated the Earth Venture program in response to recommendations made by the National Research Council (NRC) in the 2007 Decadal Survey for low-cost research and application investigations fostering innovation in Earth Science [7]. They will include stand-alone missions that use simple, small instruments, spacecraft, and launch vehicles; more complex instruments of opportunity flown on partner spacecraft and launch vehicles; or complex sets of instruments flown on suitable suborbital platforms to address focused sets of scientific questions [2]. EV-1, the first of the venture class missions, targets suborbital Earth science investigations with temporally sustained data acquisition [1].

Since the EV-1 selections, there have been two other EV selections, they are the Tropospheric Emissions: Monitoring of 
Pollution (TEMPO) [13] and Cyclone Global Navigation Satellite System (CYGNSS), which are both in formulation [14].

\section{EARTH VENTURE-1 MANAGEMENT APPROACH}

Historically, ESSP has managed space flight projects that require a relatively low risk management approach to ensure success of long duration space flight missions. In contrast, most of the investigation teams that were selected for EV-1 typically fly instruments on airborne platforms for short duration deployments that can accept greater risk because issues and challenges can be addressed between flights and deployments. EV suborbital missions are long duration investigations (multiple deployments over multiple years) that may involve multiple aircraft along with ground and space flight assets to obtain data in creative ways to further our understanding of the Earth system. Since these missions are longer in duration than traditional airborne science investigations, have a higher visibility with the sponsoring entities, and require a substantial amount of coordination and funding, additional management controls are required to ensure effective and responsible use of Agency funds and resources. These missions are composed of multiple airborne assets and if technical or programmatic challenges result in schedule delays, the resulting impacts could ripple through dependent instruments. In order to meet the needs of this new set of investigations, existing plans, procedures, and processes need to be adapted to merge current orbital and airborne paradigms.

The EV-1 management approach incorporates NPR 7120.8 [6], NASA Center practices, airborne community practices and selected aspects of NPR 7120.5 [5]. The most significant change from previous airborne missions was the addition of formal programmatic reviews, including gate reviews, but other changes included documented Program Level Requirements, documentation of the Project Plan, risk management, reserve management, tracking of schedule milestones leading up to deployments, more rigorous budget tracking and increased reporting. Each of the investigations had review requirements from the ESSP Program Office focusing on programmatic controls and risks; their respective centers focusing on technical authority issues and keeping the center informed; and aircraft reviews to ensure safety of the airplanes, crew and the public. Each of these reviews had a different focus, but each investigation was allowed to propose ways to tailor these reviews where appropriate to ensure efficient use of investigation resources. Figure 1 contrasts current ESSP PO reviews for orbital missions with airborne community reviews and how these reviews could be combined in a set of reviews for a notional EV-1 Investigation.

\subsection{A Typical Review Process}

An example of airborne community review processes and its respective nomenclature are identified in Figure 1.A and consist of an initial Airworthiness and Safety Review Board (ASRB) Review; Systems Requirements Review which is a review of the Flight Test Requirements Document; an ASRB Operational Safety Review; Flight Readiness Review; Experiment Systems Readiness Review; and a Science Team Meeting at the end of each campaign.

The ESSP Program Office review process requirements were set forth in the EV-1 NASA Research Announcement (NRA) [1], but were revised after the investigations were selected. These requirements were based on historical ESSP project life-cycle milestones (Figure 1.B), and include but are not limited to: Investigation Concept Review (ICR), and Investigation Design Review (IDR). This list of reviews constitutes the review baseline for the EV-1 investigations and is a derivative of the reviews required of NASA orbital missions (Figure 1.B). The orbital review paradigm was leveraged with current airborne practices to introduce additional rigor and definition into EV-1 formulation and implementation activities, commensurate with the increased scope of these investigations when benchmarked against historical airborne research missions.

The ICR addressed the investigation objectives, requirements, architectures, and performance measures. IDR verified that the design met the requirements within programmatic constraints (cost and schedule). IDR also ascertained whether the investigation was ready to commence with detailed design. In cases where no instrument design was needed, this detailed design refers to the design of the mission, including items such as required instruments, deployment location, deployment schedule, etc. The traditional aircraft safety and readiness reviews act as the gates to proceed toward deployed flight operations.

Complimentary to the review process requirements, the EV-1 investigations life-cycle required one Key Decision Points (KDP), where the readiness of a program/project/investigation to progress to the next phase of the life cycle was 
examined. Similar to the review baseline, the KDP required of EV-1 investigations is a subset of NASA orbital requirements (Figure 1.B). There was a required KDP-C to approve the transition from Phase B (Preliminary Design \& Technology Development) to Phase C (Final Design \& Fabrication). At the end of the investigation, an End of Prime Mission Review may be conducted to verify that all requirements were met.

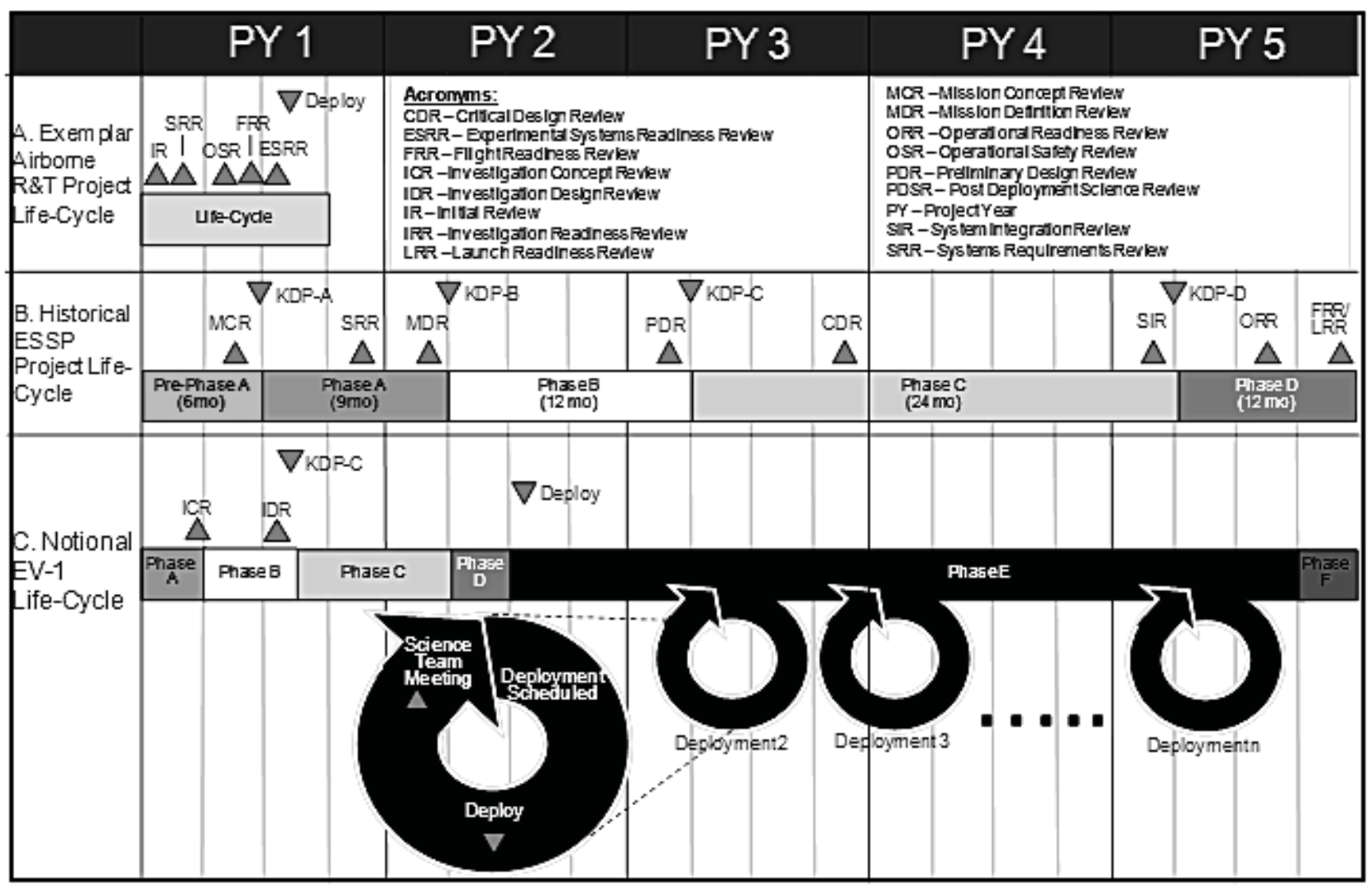

Figure 1. Typical Review Process

\subsection{A Notional Review Process}

As many as eight reviews were listed as programmatic requirements in the EV-1 solicitation [1], including System Requirements Review, Preliminary Design Review and Critical Design Review, but each investigation was encouraged to recommend tailoring to the EV-1 review baseline requirements, appropriate to the schedule, technical maturity and risk associated with each individual investigation. The option for tailoring recognizes the high technical maturity of the science instruments proposed (Technology Readiness Level 6 or higher) in conjunction with an abbreviated acquisition schedule leading up to operations. This approach also coincides with the desire by all stakeholders to optimize the time spent in the operational phase of the investigations to maximize science data collection. An example of tailoring that may be requested and negotiated with the program office is demonstrated in Figure 1.C. The adaptations depicted in Figure 1.C can be classified into four categories including: combined reviews, discretionary reviews, eliminated reviews, and supplementary reviews.

Investigations leveraging previously flown instruments in conjunction with well understood flight platforms may be able to combine IDR and ICR into a single review to expedite entry into integration and test activities. Although the focus of this paper is EV-1 requirements imposed by the program, NASA Center reviews may be combined with program reviews to streamline review activities. An example would be combining the reviews required by ESSP and the center into a single event. 
Flexible and adaptable management approaches are important to conducting the EV-1 investigations effectively and efficiently. The concept of discretionary reviews reflects the intent of the program office to work with the investigations to optimize the length of operations and science data collection, while also ensuring that adequate management controls and reviews are in place.

The eliminated reviews and KDPs in Figure 1.C should not be interpreted to mean that underlying engineering and management processes and procedures are not required, nor important. Throughout the life-cycle of each EV-1 investigation, ESSP mission managers, Headquarters personnel, Center personnel and flight platform stakeholders will be working in close and constant coordination with the EV-1 investigation teams to ensure necessary technical and programmatic oversight. The elimination of reviews and KDPs in this notional scenario acknowledges that in some cases, day-to-day interactions and less formal status reviews are sufficient when taking into consideration the heritage and maturity of investigation technologies and operational concepts and to facilitate rapid deployment following award as required for some of the EV-1 investigations.

The collection of temporally-sustained science data via multiple airborne deployments allows for modification and calibration of instruments between flights and deployments. In cases where substantive changes occur to the instrument or its integration with the flight platform, a supplementary review ( $\triangle \mathrm{IDR}$ ) can be added to the review cycle in figure 1 even though the investigation is in the operational phase. The occurrence of design reviews during the operational phase is uncommon when contrasted against historical project life-cycles. Yet, this practice enables EV-1 designs to successfully evolve during the operational phase while also ensuring adequate review has transpired for any significant modifications. Another supplementary review is portrayed by the Science Team Meeting at the end of each deployment. The Science Team Meetings allow the investigation teams to review the science data collected in partnership with program and project scientists and collect lessons learned to improve future deployments.

These formal reviews were useful to management by highlighting concerns, issues, and risks that were not previously identified by the investigation. This allowed management an opportunity to understand and act on concerns to prevent future problems.

\subsection{Program Level Requirements}

The EV-1 investigations were required to develop program level requirements that were approved by the NASA HQ Earth Science Division. These requirements documented which measurements would be taken along with the precision and duration of flights. It is important to point out that there are two levels of requirements - baseline and threshold and the project can go through a descope process to deliver less than the baseline but still be considered successful. These requirements ensured all parties have the same understanding of what the investigation plans on doing for the duration of the life cycle. Documenting these requirements was helpful to HQ management and was also helpful to the investigations. The documentation of these requirements benefited HQ management by allowing them to understand specifically what the investigation plannned to accomplish and allows HQ to measure performance of the investigation. The documentation of these requirements also helps the investigations by ensuring NASA does create conflicts outside of the investigation to impact the investigation's ability to meet the requirements. There were many cases where it did not appear the program level requirements would be met because of outside influences on the investigation. With the documented requirements, this brought the proper attention to the problem and in some cases the outside influences were adjusted to allow the investigation to complete its program level requirements. To illustrate, in response to competition for a critical NASA airborne asset, HQ provided funding outside of EV-1 to modify a second aircraft to meet program needs. There were also cases where the program level requirements were adjusted, but by documenting these requirements and the need for a formal change, it was clear to all parties the reason for the change in requirements.

\subsection{Reporting and Project Plan}

The EV-1 Investigations were required to document the investigation processes in a Project Plan and provide monthly reports against that plan to the ESSP Program Office. The monthly reports include accomplishments, issues/concerns, schedule plan versus actual, budget plan versus actual, reserve status, risks and mitigation status and any science results. This reporting allowed the ESSP Program Office and HQ management to stay informed on status and issues for these high visibility investigations. Along with the monthly reports there are regular conference calls between the 
investigation and the ESSP Program Office to update status. The Project Plan captured the approach for how the project would be managed and included baseline schedule milestones, baseline budget, performance tracking process, risk management process, reserve management process, configuration control and many other aspects of project management.

The management processes used for EV-1 are significantly modified from those applied orbital missions. Some examples of differences are in the areas of schedule and documentation. For the schedule area, a significant difference is that on EV-1, the investigations were required to report against a set of milestones rather than a detailed schedule. In regards to documentation, orbital missions require formal documentation whereas on EV-1 many elements were documented using presentation charts to reduce workload and proved sufficient to the lower complexity of the investigations.

By tailoring the reporting requirements and the management plan, ESSP Program Office management is able to track the progress of the investigation and gain confidence in their success and easily relay that information up to higher level management while not over burdening the investigations.

\section{FUTURE EARTH VENTURE SUBORBITAL MISSIONS}

Recently, the suborbital EV activites were renamed Earth Venture Suborbital (EVS). The Earth Venture Suborbital (EVS) selections are recurring with new selections being made every four years. In late June 2013, the EVS-2 solicition was publicized [15], seeking the next round of EVS proposals in January 2014.

Based on lessons learned from EV-1, the management approach for future EVS missions will be tailored. As part of this evolution, most of the differences will be in the area of reviews. There will be a KDP-C, or Investigation Confirmation Review, to obtain approval from HQ ESD to proceed into implementation, but the prior independent reviews that were held for EV-1 will not be conducted. These reviews were performed in EV-1 to prepare the Investigation for the KDP-C, but for EVS-2 investigations the ESSP Program Office will provide informal guidance, rather than formal independent reviews, leading up the KDP-C/Investigation Confirmation Review to facilitate a successful review.

The investigations will still use milestones to track progress on schedule and will still be required to develop a Project Plan to describe how that project will be managed. Most of the elements in the Project Plan will still be required but the formal documentation of some of these elements may be relaxed. Monthly reporting and regular conference calls will still be needed to allow ESSP Program Office to follow the progress of the investigations. The EVS investigations will also have requirements that will be approved by ESD, which will document the baseline and threshold requirements that the investigation must satisfy.

\section{SUMMARY}

The Earth Venture class of missions is a welcomed addition to NASA SMD's portfolio of science missions charged with advancing the fundamental knowledge of the global integrated Earth system. The first of these lower cost research and application missions, EV-1, will reap the scientific rewards that originate from long-term temporal satellite missions by utilizing technical maturity and an agile data acquisition process enjoyed by the airborne science community. This new approach to Earth system science demands an accompanying management approach that accommodates the programmatic rigor necessary for sustained mission success with a flexible process appropriate for risk-tolerant investigations with mature technology. The ESSP Program Office is excited to assume responsibility for the management of the EV missions and to set a successful precedent for future airborne investigations with EV-1. Future EVS Investigations will use similar management processes that were used on EV-1, but will be relaxed in the area of reviews. 


\section{REFERENCES}

[1] New proposal opportunity in Appendix A.39: ESSP Venture-class Science Investigations: Earth Venture-1 and Appendix A.39: ESSP Venture-class Science Investigations: Earth Venture-1 from Research Opportunities in Space and Earth Sciences (ROSES) 2009. Solicitation: NNH09ZDA001N, 2009.

[2] Allen, B.D.; Braun, S.A.; Crawford, J.H.; Jensen, E.J.; Miller, C.E.; Moghaddam, M.; Maring, H., "Proposed investigations from NASA's Earth Venture-1 (EV-1) airborne science selections," Proc. IGARSS, 2010 IEEE International , 2575-2578, (2010).

[3] Systems Engineering Handbook, NASA/SP-2007-6105, Rev1. Washington, DC, 2007.

[4] Science Mission Directorate (SMD) Management Handbook. Washington, DC, 2008.

[5] NPR 7120.5, NASA Space Flight Program and Project Management Processes and Requirements. Washington, DC, 2007.

[6] NPR 7120.8, NASA Research and Technology Program and Project Management Requirements. Washington, DC, 2008.

[7] Earth Science and Applications from Space: National Imperatives for the Next Decade and Beyond, Committee on Earth Science and Applications from Space: A Community Assessment and Strategy for the Future, National Research Council, 2007.

$[8]<$ http://www.csr.utexas.edu/grace $>$

[9] < http://cloudsat.atmos.colostate.edu/overview $>$

[10] <http://www-calipso.larc.nasa.gov/>

[11] <http://aquarius.nasa.gov/>

[12] <http://oco.jpl.nasa.gov/>

[13] <http://science1.nasa.gov/missions/tempo/>

[14] <http://aoss-research.engin.umich.edu/missions/cygnss/>

[15] New proposal opportunity in Appendix A.30: ESSP Venture-class Science Investigations: Earth Venture-1 and Appendix A.39: ESSP Venture-class Science Investigations: Earth Venture Suborbital-2 from Research Opportunities in Space and Earth Sciences (ROSES) - 2009 . Solicitation: NNH13ZDA001N-EVS2, 2013. <http://nspires.nasaprs.com> 